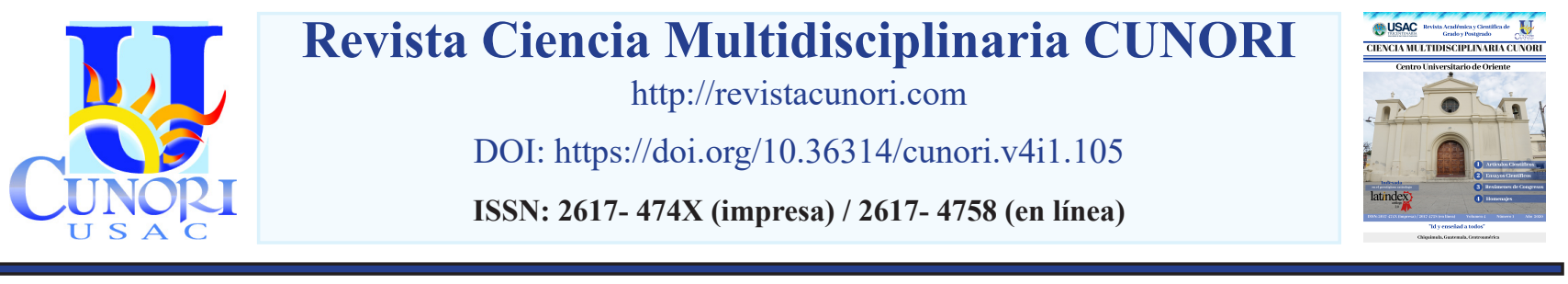

Como citar el artículo

Mateo, S., Monterroso, L. y Retana, R. (2020). Ácido úrico y su relación con injuria renal aguda. Revista Ciencia Multidisciplinaria CUNORI 4(1). 15-20. DOI: https://doi.org/10.36314/cunori.v4i1.105

\title{
Ácido úrico y su relación con injuria renal aguda
}

\section{Uric acid and its relationship with acute renal injury}

\author{
Shirley Mateo, Luis Monterroso \& Ronaldo Retana \\ Centro Universitario de Oriente (CUNORI), Universidad de San Carlos de Guatemala
}

Recibido: 30 de junio de 2019 / Revisión: 05 de julio de 2019 / Aceptado: 06 de febrero de 2020

Disponible en internet el 29 de mayo de 2020

\section{Resumen}

*Autor para correspondencia.

Correo electrónico: shirnethmateo@hotmail.com

$\mathrm{E}$ 1 ácido úrico, el producto final del metabolismo de las purinas, se excreta predominantemente en los túbulos proximales.

Recientemente, se ha informado de que los niveles anormales de ácido úrico es un factor de riesgo para la enfermedad renal (Otomo et al. 2016).Este estudio analítico se centró en determinar la relación de ácido úrico alterado con injuria renal aguda en pacientes ingresados a los servicios de medicina interna en el Hospital Nacional de Chiquimula en los meses de enero del 2017 a diciembre del año 2018. De los 267 pacientes a quienes se les realizó niveles de ácido úrico al ingreso hospitalario el 71\% desarrollaron algún estadio de injuria renal aguda con valores mayores o igual a $7 \mathrm{mg} / \mathrm{dL}$, obteniendo un Odds Ratio de 4.4062 , riesgo relativo 1.4416 , con un intervalo de confianza del $95 \%$, con valor p de 0.000 siendo altamente significativo. La injuria renal aguda se desarrolló más frecuentemente en estadio II, con niveles de ácido úrico $7 \mathrm{mg} / \mathrm{dL}$ en el $31 \%$ de los casos. En estadio I con niveles de ácido úrico $5 \mathrm{mg} / \mathrm{dL}$ en el $8 \%$ y solamente en estadio III con valores de ácido úrico $8 \mathrm{mg} / \mathrm{dL}$ en el 7\% de los casos. Las principales características clínicas de los pacientes que desarrollaron injuria renal a expensas de ácido úrico alterado fueron: género femenino con $53 \%$, el rango de edad más afectado de 51 a 60 años con el 31\% y el lugar de procedencia más afectado fue el departamento de Chiquimula con el 41\%. De las comorbilidades asociadas de los pacientes que desarrollaron injuria renal aguda por ácido úrico alterado, la principal es hipertensión arterial con el 28\%, seguido por diabetes mellitus II con el 25\%, en la mayoría de los casos.

Palabras clave: ácido úrico, injuria renal aguda

\section{Abstract}

$\mathrm{U}$ ric acid, the final product of purine metabolism, is excreted predominantly in the proximal tubules. Recently, it has been reported that abnormal levels of uric acid is a risk factor for kidney disease (Otomo et al. 2016). This analytical study focused on determining the relationship of altered uric acid with acute renal injury in patients admitted to internal medicine services at the Hospital Nacional de Chiquimula from January 2017 to December 2018. Of the 267 patients at those who had uric acid levels at hospital admission, 71\% developed some stage of acute renal injury with values greater than or equal to $7 \mathrm{mg} / \mathrm{dL}$, obtaining an Odds Ratio of 4.4062, relative risk 1.4416, with a confidence interval of $95 \%$. \%, with p-value of 0.000 being highly significant. Acute renal injury developed more frequently in stage II, with uric acid levels $7 \mathrm{mg} / \mathrm{dL}$ in $31 \%$ of cases. In stage I with uric acid levels $5 \mathrm{mg} / \mathrm{dL}$ in $8 \%$ and only in stage III with uric acid values $8 \mathrm{mg} / \mathrm{dL}$ in $7 \%$ of cases. The main clinical characteristics of the patients who developed renal injury at the expense of altered uric acid were: female gender with 53\%, the most affected age range of 51 to 60 years with $31 \%$ and the place of origin most affected were the department of Chiquimula with $41 \%$. Of the associated 
comorbidities of patients who developed acute renal injury due to altered uric acid, the main one is arterial hypertension with $28 \%$, followed by diabetes mellitus II with $25 \%$, in the majority of cases.

Keywords: uric acid, acute renal injury

\section{Introducción}

El ácido úrico (AU) es el producto final del catabolismo de las purinas, bases nitrogenadas constituyentes de los ácidos nucleicos. La producción endógena de AU se da principalmente en el hígado, los intestinos y otros tejidos como los músculos, los riñones y el endotelio vascular (Carvajal 2016). Injuria renal aguda (Acute Kidney Injury AKI) se define como "una abrupta reducción de la función renal, en un período de 48 horas" (IGSS 2017). Los criterios actuales para el diagnóstico y la estadificación de lesión renal aguda propusieron una definición única para esta, que debería ser útil para la práctica clínica, la investigación y la salud pública. Definiendo lesión renal aguda como cualquiera de los siguientes enunciados:

- Aumento de la creatinina sérica $0.3 \mathrm{mg} / \mathrm{dL}$ (26.5 mmol/l) en 48 horas.

- Aumento de la creatinina sérica $>1.5$ veces el valor basal, que se sospeche se haya producido dentro de los 7 días previos.

- Volumen de orina menor de $0.5 \mathrm{ml} / \mathrm{kg} /$ hora durante 6 horas. (Toro et al. 2015).

El ácido úrico, el producto final del metabolismo de las purinas, se excreta predominantemente en los túbulos proximales (EMPENDIUM, 2018). Recientemente, se ha informado de que los niveles anormales de ácido úrico en suero (SUA) son un factor de riesgo para la enfermedad cardiovascular, enfermedad renal crónica, síndrome metabólico. Ejaz et al. también informó que un alto valor SUA es un factor de riesgo independiente para la IRA, y que la reducción de los niveles de SUA podría prevenir IRA. (Otomo et al. 2016).

\section{Materiales y métodos}

Para el estudio se tomó una muestra de 267 pacientes ingresados a los servicios de medicina interna en los meses de enero del 2017 a diciembre del 2018 en el Hospital Nacional de Chiquimula. Para la recolección de datos fue utilizada una boleta de recolección de datos, dividida en tres partes, la primera parte recopiló características epidemiológicas tales como sexo, edad, lugar de procedencia. La segunda el valor de ácido úrico, creatinina, excreta urinaria y el estadio de injuria renal aguda. Y la última parte las comorbilidades asociadas. Los datos obtenidos se ingresaron y se tabularon utilizando estadística analítica, mostrando los resultados mediante tablas de información. 


\section{Resultados}

Tabla 1. Relación del ácido úrico alterado e injuria renal aguda en los pacientes ingresados a los servicios de Medicina Interna del Hospital Nacional de Chiquimula, en los meses de enero del 2017 a diciembre del 2018.

\begin{tabular}{cccc}
\hline & Con injuria renal aguda & Sin injuria renal aguda \\
Ácido úrico alterado & 94 casos & 14 casos \\
Ácido úrico normal & 96 casos & 63 casos \\
\hline & & \\
\hline & IC 95\% & \\
Odds Ratio & 4.4063 & Inferior & Superior \\
Riesgo relativo & 1.4416 & 2.31 & 8.39 \\
Valor P & 0.000 & 1.24 & 1.66 \\
\hline
\end{tabular}

Boleta de recolección de datos, 2019

Se obtuvo un Odss Ratio de 4.4063 ( $<1$ valor esperado) significando que es un factor determinante a largo plazo, riesgo relativo 1.4416 el riesgo de desarrollar injuria renal por los niveles de ácido úrico alterados es a corto plazo, con intervalo de confianza de $95 \%$, obteniendo un valor p 0.000 , siendo altamente significativo la relación de ácido úrico alterado con el desarrollo de injuria renal aguda.

Tabla 2. Correlación del valor de ácido úrico alterado y el estadio de injuria renal aguda en los pacientes ingresados a los servicios de Medicina Interna del Hospital Nacional de Chiquimula, en los meses de enero del 2017 a diciembre del 2018.

\begin{tabular}{|c|c|c|c|c|}
\hline & $5 \mathrm{mg} / \mathrm{dL}$ & $6 \mathrm{mg} / \mathrm{dL}$ & $7 \mathrm{mg} / \mathrm{dL}$ & $8 \mathrm{mg} / \mathrm{dL}$ \\
\hline Estadio I & $\begin{array}{c}15 \text { pacientes } \\
(8 \%)\end{array}$ & $\begin{array}{c}11 \text { pacientes } \\
(6 \%)\end{array}$ & $\begin{array}{c}14 \text { pacientes } \\
(7 \%)\end{array}$ & $\begin{array}{c}4 \text { pacientes } \\
(2 \%)\end{array}$ \\
\hline Estadio II & $\begin{array}{c}18 \text { pacientes } \\
(9 \%)\end{array}$ & $\begin{array}{c}43 \text { pacientes } \\
(23 \%)\end{array}$ & $\begin{array}{c}58 \text { pacientes } \\
(31 \%)\end{array}$ & 0 pacientes \\
\hline Estadio III & 0 pacientes & $\begin{array}{c}9 \text { pacientes } \\
(5 \%)\end{array}$ & $\begin{array}{c}4 \text { pacientes } \\
(2 \%)\end{array}$ & $\begin{array}{c}14 \text { pacientes } \\
(7 \%)\end{array}$ \\
\hline & 33 & 63 & 76 & 18 \\
\hline
\end{tabular}

Boleta de recolección de datos, 2019 
La injuria renal aguda fue más frecuentemente en estadio II con ácido úrico $7 \mathrm{mg} / \mathrm{dL}$ en el 31\% (58), ácido úrico $6 \mathrm{mg} / \mathrm{dL}$ el 23\% (43) y $5 \mathrm{mg} / \mathrm{dL}$ con $9 \%$ (18). Seguido por injuria renal aguda estadio I, más frecuentemente desarrollada por ácido úrico $5 \mathrm{mg} / \mathrm{dL}$ en el $8 \%(15)$ y ácido úrico $7 \mathrm{mg} / \mathrm{dL}$ en el 7\% (14). En el estadio III ácido úrico $8 \mathrm{mg} / \mathrm{dL}$ con 7\% (14) y niveles de ácido úrico 6mg/dl con 5\% (9) de los casos.

Tabla 3. Características de los pacientes que desarrollaron injuria renal por ácido úrico ingresados a los servicios de Medicina Interna del Hospital Nacional de Chiquimula, en los meses de enero del 2017 a diciembre del 2018.

\begin{tabular}{|c|c|c|}
\hline Características Generales & $\mathbf{N}$ & $\%$ \\
\hline \multicolumn{3}{|l|}{ Sexo } \\
\hline Masculino & 89 & 47 \\
\hline Femenino & 101 & 53 \\
\hline \multicolumn{3}{|l|}{ Edad } \\
\hline $31-40$ & 18 & 9 \\
\hline $41-50$ & 51 & 27 \\
\hline $51-60$ & 57 & 31 \\
\hline $61-69$ & 46 & 24 \\
\hline mayor de 70 & 18 & 9 \\
\hline \multicolumn{3}{|l|}{ Procedencia } \\
\hline Chiquimula urbana & 42 & 22 \\
\hline Chiquimula rural & 36 & 19 \\
\hline Jutiapa & 53 & 28 \\
\hline Jalapa & 21 & 11 \\
\hline Izabal & 18 & 9 \\
\hline Petén & 13 & 7 \\
\hline El progreso & 7 & 4 \\
\hline \multicolumn{3}{|l|}{ Comorbilidades } \\
\hline Hipertensión arterial & 54 & 29 \\
\hline Diabetes mellitus II & 47 & 25 \\
\hline Neumonía adquirida en la comunidad & 22 & 13 \\
\hline Evento cerebro vascular & 14 & 8 \\
\hline Shock séptico & 12 & 7 \\
\hline Infarto agudo al miocardio & 10 & 5 \\
\hline Cirrosis hepática & 9 & 3 \\
\hline Cetoacidosis diabética & 8 & 2 \\
\hline
\end{tabular}

Boleta de recolección de datos, 2019. 
El 53\% desarrolló injuria renal aguda por ácido úrico alterado es el sexo femenino y 47\% es masculino. El 31\% pertenecen al rango de edad de 51-60 años, seguido el 27\% (51) con 41-50 años, 24\% (46) con 61-69 años, 9\% (17) en los rangos 31-40 y mayor a 70 años. El 41\% de los pacientes pertenecen al departamento de Chiquimula, de los cuales el 22\% pertenecen al área urbana, 19\% al área rural, 28\%proceden de Jutiapa, 11\% al departamento de Jalapa, 9\% al departamento de Izabal, 7\% al departamento de Petén y 4\% pertenecen al departamento de El Progreso.

\section{Discusión}

De un total de 267 pacientes sometidos al estudio, el 71\% desarrolló algún estadio de injuria renal aguda por valores de ácido úrico mayor o igual a $7 \mathrm{mg} / \mathrm{dL}$, por lo que se rechaza la hipótesis nula, debido a que existe un valor de p altamente significativo de 0.000 y Odds Ratio de 4.4062. La injuria renal aguda se desarrolló más frecuentemente en estadio II, con niveles de ácido úrico $7 \mathrm{mg} / \mathrm{dL}$ en el $31 \%$ de los casos. En estadio I con niveles de ácido úrico $5 \mathrm{mg} / \mathrm{dL}$ en el $8 \%$ y solamente en estadio III con valores de ácido úrico $8 \mathrm{mg} / \mathrm{dL}$ en el $7 \%$ de los casos. Las principales características clínicas de los pacientes que desarrollaron injuria renal aguda a expensas de ácido úrico elevado fueron: sexo femenino 53\%, el rango de edad más frecuentemente afectado es de 51 a 60 años con el 31\%, y el lugar de procedencia más frecuente fueron del departamento de Chiquimula con $41 \%$.Se determinó que las comorbilidades asociadas de los pacientes que desarrolló injuria renal aguda por ácido úrico alterado fueron; hipertensión arterial con 29\%, seguido el 25\% diabetes mellitus en la mayoría de los casos.

\section{Agradecimientos}

Al Comité Organizador de Trabajos de Graduación de Medicina por aprobar el tema a investigar. Al Dr. Luis Jovito Monterroso Valdés, maestro en ciencias médicas en especialidad en medicina interna y especialidad en nefrología, por su asesoría y dedicación brindada durante la realización de la investigación. Al Dr. Edvin Danilo Mazariegos Albanés, especialista en medicina interna, por su apoyo en la revisión del presente manuscrito.

\section{Referencias}

Carvajal, C. 2016. El ácido úrico: de la gota y otros males, 33(1), 1-8. Recuperado de: en http://www. scielo.sa.cr/pdf/mlcr/v33n1/1409-0015-mlcr-33-01-00182.pdf

EMPENDIUM (Unión Europea). 2018. Lesión renal aguda, In Manual MIBE. Recuperado de: en https:// empendium.com/manualmibe/chapter/B34.II.14.1.

IGSS (Instituto Guatemalteco de Seguridad Social). 2017. Manejo de la lesión renal aguda, 40 p. Recuperado de: en https://www.igssgt.org/images/gpc-be/medicina_interna/GPC-BE-No-34-Manejode-la-Lesion-Renal-Aguda.pdf

Otomo, K. 2016. La concentración sérica de ácido úrico como factor de riesgo de lesión renal aguda en pacientes hospitalizados: un análisis retrospectivo de la base de datos utilizando el sistema integrado 
de información médica en el Hospital Escuela de Medicina de Kochi, 20(2), 235-243. Recuperado de: en doi 10.1007 / s10157-015-1156-5

Toro, L. 2015. Biomarcadores de injuria renal aguda, 30(2), 67-74. Recuperado de: en https://www.medicina-intensiva.cl/revistaweb/revistas/indice/2015-2/pdf/4.pdf.

\section{Sobre la autora}

\section{Shirley Ninneth Mateo Galicia}

Es Médico y Cirujano del Centro Universitario de Oriente - CUNORI- de la Universidad San Carlos de Guatemala - USAC, actualmente es residente de Medicina Interna del Instituto Guatemalteco de Seguridad Social -IGSS-.

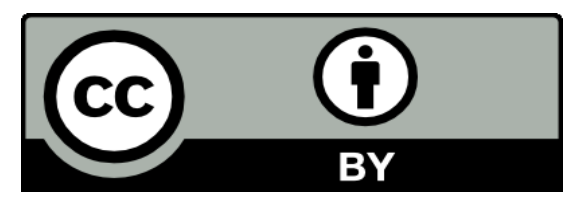

Este texto está protegido por una licencia CreativeCommons 4.0.

Usted es libre para compartir, copiar y redistribuir el material en cualquier medio o formato y adaptar el documento, remezclar, transformar y crear a partir del material para cualquier propósito, incluso comercialmente, siempre que cumpla la condición de atribución: usted debe reconocer el crédito de una obra de manera adecuada, proporcionar un enlace a la licencia, e indicar si se han realizado cambios. Puede hacerlo en cualquier forma razonable, pero no de forma tal que sugiera que tiene el apoyo del licenciante o lo recibe por el uso que hace. 